\title{
The Stability of Two-Step Runge-Kutta Methods for Neutral Delay Integro Differential-Algebraic Equations with Many Delays
}

\author{
Haiyan Yuan ${ }^{1,2}$ and Jihong Shen ${ }^{1}$ \\ ${ }^{1}$ College of Automation, Harbin Engineering University, Harbin 150001, China \\ ${ }^{2}$ Department of Mathematics, Heilongjiang Institute of Technology, Harbin 150050, China \\ Correspondence should be addressed to Jihong Shen; shenjihong@hrbeu.edu.cn
}

Received 3 January 2014; Revised 22 April 2014; Accepted 22 April 2014; Published 9 June 2014

Academic Editor: J. A. Tenreiro Machado

Copyright ( $\odot 2014 \mathrm{H}$. Yuan and J. Shen. This is an open access article distributed under the Creative Commons Attribution License, which permits unrestricted use, distribution, and reproduction in any medium, provided the original work is properly cited.

This paper studies the asymptotic stability of the two-step Runge-Kutta methods for neutral delay integro differential-algebraic equations with many delays. It proves that A-stable two-step Runge-Kutta methods are asymptotically stable for neutral delay integro differential-algebraic equations with many delays.

\section{Introduction}

The stability of numerical methods for delay differential equations has been intensively studied in [1-3] for many years. These equations appeared in a wide variety of scientific and engineering fields, such as circuit analysis, computeraided design power systems, and optimal control. The structure for these, the order of convergence, and the asymptotic stability of numerical methods have been studied in [46]. Zhu and Petzold investigated the asymptotic stability of neutral delay differential equations with $\theta$-methods, RungeKutta methods, BDF methods, and linear multistep methods [7]. Zhao et al. studied the stability of neutral delay differential equations with Rosenbrock methods [8]. Yu et al. studied the general neutral delay differential equations with multistep methods [9]. More recently, there is a growing interest in the analysis of delay integro differential equations. Baker and Ford [10] studied the asymptotic stability of a class of linear multistep (LM) methods for scalar linear delay integro differential equations; Koto [11] dealt with the linear stability of Runge-Kutta (R-K) methods for systems of delay integro differential equations; Huang and Vandewalle [12] gave sufficient and necessary stability conditions for exact and discrete solutions of linear scalar delay integro differential equations, and Luzyanina et al. [13] developed computational procedures for determining the stability of delay integro differential equations. Zhang and Vandewalle [14] gave the stability criteria for exact and discrete solution of neutral multidelay integro differential equations. Although the stability of numerical methods for delay integro differential equations has been very intensively studied, the stability of delay integro differential equations with many delays has not been studied so far.

In this paper, we focus on the asymptotic stability of numerical methods for neutral delay integro differentialalgebraic equations with many delays. This paper is structured as follows. In Section 2 we give asymptotic stability of the analytical solution and introduce two-step Runge-Kutta methods and the stability region. In Section 3, we deal with the asymptotic stability of two-step Runge-Kutta method for neutral delay integro differential-algebraic equations with many delays; the theoretical results are proved. In Section 4, an example is given to illustrate the theoretical results.

\section{Asymptotic Stability of the Analytical Solution}

2.1. Asymptotic Stability of the Analytical Solution of Neutral Delay Integro Differential-Algebraic Equation with Many Delays. In this section, we consider the following linear 
system:

$$
\begin{gathered}
A u^{\prime}(t)+B u(t)+\sum_{q=1}^{m} C_{q} u^{\prime}\left(t-\tau_{q}\right)+\sum_{q=1}^{m} D_{q} u\left(t-\tau_{q}\right) \\
+\sum_{q=1}^{m} G_{q} \int_{t-\tau_{q}}^{t} u(\delta) d \delta=0, \quad t \geq 0, \\
u(t)=\varphi(t), \quad t \in[-\tau, 0),
\end{gathered}
$$

where $A, B, C_{q}, D_{q}, G_{q} \in R^{d \times d}, A$ is a singular matrix, $\tau_{q}$ is a given positive delay constant $(q=1,2, \ldots, m)$, and $0<$ $\tau_{1} \leq \tau_{2} \leq \cdots \leq \tau_{m}=\tau . \varphi(t)$ denotes a given vector-valued function and $u(t)$ is a vector-valued unknown function to be solved for $t \geq 0$.

In order to obtain the characteristic equation of system (1), we focus on the exponential solutions $u(t)=e^{s t} x$ of (1); here $x=\left(x_{1}, x_{2}, \ldots, x_{d}\right)^{\mathrm{T}} \in C^{d}$ denotes the unknown vector. Then we have

$$
\begin{gathered}
u(t)=\left(e^{s t} x_{1}, e^{s t} x_{2}, \ldots, e^{s t} x_{d}\right)^{\mathrm{T}}, \\
u^{\prime}(t)=s e^{s t} x, \\
u\left(t-\tau_{q}\right)=\left(e^{s\left(t-\tau_{q}\right)} x_{1}, e^{s\left(t-\tau_{q}\right)} x_{2}, \ldots, e^{s\left(t-\tau_{q}\right)} x_{d}\right)^{\mathrm{T}}, \\
q=1,2, \ldots, m, \\
u^{\prime}\left(t-\tau_{q}\right)=s e^{s\left(t-\tau_{q}\right)} x, \quad q=1,2, \ldots, m .
\end{gathered}
$$

Substituting the above results into (1), we have the following equation:

$$
\begin{gathered}
{\left[s A+B+s \sum_{q=1}^{m} C_{q} e^{-s \tau_{q}}+\sum_{q=1}^{m} D_{q} e^{-s \tau_{q}}\right.} \\
\left.+s^{-1} \sum_{q=1}^{m} G_{q}\left(1-e^{-s \tau_{q}}\right)\right] x=0 .
\end{gathered}
$$

The existence of a nonzero $x$ in (2e) implies the characteristic equation of system (1) holds; that is, the following equation holds:

$$
\begin{aligned}
\operatorname{det}[s A & +B+s \sum_{q=1}^{m} C_{q} e^{-s \tau_{q}} \\
& +\sum_{q=1}^{m} D_{q} e^{-s \tau_{q}} \\
& \left.+s^{-1} \sum_{q=1}^{m} G_{q}\left(1-e^{-s \tau_{q}}\right)\right]=0 .
\end{aligned}
$$

Definition 1 (see [13]). Equation (1) is said to be asymptotically stable, if for any continuous differential initial function and for any delay $\tau_{q}>0, q=1,2, \ldots, m$ the analytical solution to (1) satisfies $\lim _{t \rightarrow \infty} u(t)=0$.
We know that the stability of analytical solution can be studied via the characteristic equation, so we give a criterion for the asymptotic stability of (1), which is based on the following lemmas.

Lemma 2 (see [14]). Assume

$$
\operatorname{Sup}\{\operatorname{Re}(\lambda): p(\lambda)=0\}<0,
$$

where $p(\lambda)=\operatorname{det}\left\{\lambda A+B+\lambda \sum_{q=1}^{m} C_{q} e^{-\lambda \tau_{q}}+\sum_{q=1}^{m} D_{q} e^{-\lambda \tau_{q}}+\right.$ $\left.\sum_{q=1}^{m} G_{q} \tau_{q} \eta\left(e^{-\lambda \tau_{q}}\right)\right\}$ is the characteristic polynomial of (1). Then, system (1) is asymptotically stable.

Where $\eta(z)$ is a complex function defined by

$$
\eta(z)= \begin{cases}\frac{1-z}{\ln z}, & z \in C \backslash\{0,1\} \\ 0, & z=0 \\ -1, & z=1 .\end{cases}
$$

And $\ln z=\ln |z|+i \arg z(z=0,1 ;-\pi<\arg z \leq \pi)$ is the principal branch of the multivalued complex natural logarithm.

Lemma 3 (see [14]). Function $\eta(z)$ is analytic in $C \backslash R_{0}^{-}$and satisfies $|\eta(z)| \leq 1$ for $|z| \leq 1$, where $R_{0}^{-}=\{x \in R: x \leq 0\}$.

Lemma 4. If the matrix $\left(A+\sum_{q=1}^{m} C_{q} e^{-\lambda \tau_{q}}\right)$ is invertible for $\operatorname{Re}(\lambda) \geq r$, where $r \in R$, then the function

$$
\begin{aligned}
\widetilde{p}(\lambda)=\operatorname{det}\left\{\lambda^{2} I_{d}+\left(A+\sum_{q=1}^{m} C_{q} e^{-\lambda \tau_{q}}\right)^{-1}\right. \\
\times\left(\lambda B+\lambda \sum_{q=1}^{m} D_{q} e^{-\lambda \tau_{q}}\right. \\
\left.\left.+\sum_{q=1}^{m} G_{q}\left(1-e^{-\lambda \tau_{q}}\right)\right)\right\}
\end{aligned}
$$

has at most a finite number of zeros for $\operatorname{Re}(\lambda) \geq r$.

Proof. When $\operatorname{Re}(\lambda) \geq r$, the function $\widetilde{p}(\lambda)$ can be expanded into the following form:

$$
\begin{aligned}
\widetilde{p}(\lambda)= & \lambda^{2 d}+\psi_{2 d-1}\left(e^{-\lambda \tau_{1}}, e^{-\lambda \tau_{2}}, \ldots, e^{-\lambda \tau_{m 1}}\right) \lambda^{2 d-1} \\
& +\cdots+\psi_{0}\left(e^{-\lambda \tau_{1}}, e^{-\lambda \tau_{2}}, \ldots, e^{-\lambda \tau_{m 1}}\right)
\end{aligned}
$$

where $\psi_{i}\left(e^{-\lambda \tau_{1}}, e^{-\lambda \tau_{2}}, \ldots, e^{-\lambda \tau_{m 1}}\right), i=0,1, \ldots, 2 d-1$, are rational functions for the expressions $e^{-\lambda \tau_{1}}, e^{-\lambda \tau_{2}}, \ldots, e^{-\lambda \tau_{m 1}}$, and they have no poles for $\operatorname{Re}(\lambda) \geq r$.

Since $\tau_{i}>0$, we have that

$$
\left|e^{-\lambda \tau_{i}}\right|=e^{-\tau_{i} \operatorname{Re}(\lambda)} \leq e^{-\tau_{i} r}, \quad \text { for } \operatorname{Re}(\lambda) \geq r .
$$

Hence, there exist constants $K_{i}>0$ such that

$$
\left|\psi_{i}\left(e^{-\lambda \tau_{1}}, e^{-\lambda \tau_{2}}, \ldots, e^{-\lambda \tau_{m 1}}\right)\right| \leq K_{i}, \quad i=0,1, \ldots, 2 d-1 .
$$


Let $M$ be a positive number large enough such that

$$
\frac{K_{2 d-1}}{M}+\frac{K_{2 d-2}}{M^{2}}+\cdots+\frac{K_{0}}{M^{2 d}}<1,
$$

which implies that, for $\operatorname{Re}(\lambda) \geq r$ and $|\lambda| \geq M$,

$$
|\widetilde{p}(\lambda)| \geq|\lambda|^{2 d}\left[1-\frac{K_{2 d-1}}{M}-\frac{K_{2 d-2}}{M^{2}}-\cdots-\frac{K_{0}}{M^{2 d}}\right]>0 .
$$

That is, $\widetilde{p}(\lambda) \neq 0$ in the set $\{\lambda: \operatorname{Re} \lambda \geq r,|\lambda| \geq M\}$.

By the isolation property of the zeros for analytic functions, $\widetilde{p}(\lambda)$ has at most a finite number of zeros in the set $\{\lambda: \operatorname{Re} \lambda \geq r,|\lambda|<M\} ;$ this proves the lemma.

In the following, we denote the spectrum of a square matrix $A$ by $\sigma(A)$ and introduce the set

$$
C^{-}=\{z \in C: \operatorname{Re}(z)<0\}
$$

Theorem 5. System (1) is asymptotically stable if the following conditions are satisfied:

(a) $\operatorname{det}\left(A+\sum_{q=1}^{m} \xi_{q} C_{q}\right) \neq 0$ for $\left|\xi_{q}\right| \leq 1$,

(b) $\sigma(G(\xi)) \subseteq C^{-}$for $\xi=\left(\xi_{1}, \xi_{2}, \ldots, \xi_{m}\right)^{\mathrm{T}}$ with $\left|\xi_{q}\right| \leq 1$, where

$$
\begin{aligned}
G(\xi)= & \left(A+\sum_{q=1}^{m} \xi_{q} C_{q}\right)^{-1} \\
& \times\left(-B-\sum_{q=1}^{m} \xi_{q} D_{q}-\sum_{q=1}^{m} \eta\left(\xi_{q}\right) G_{q} \tau_{q}\right) .
\end{aligned}
$$

Proof. When $\left|\xi_{q}\right| \leq 1, q=1,2, \ldots, m$, condition (a) leads to

$$
\begin{gathered}
\widehat{P}\left(\lambda, \xi_{1}, \xi_{2}, \ldots, \xi_{m}\right) \\
=\operatorname{det}\left(\lambda A+\lambda \sum_{q=1}^{m} \xi_{q} C_{q}+B\right. \\
+\sum_{q=1}^{m} \xi_{q} D_{q} \\
\left.+\sum_{q=1}^{m} \eta\left(\xi_{q}\right) G_{q} \tau_{q}\right) \\
=\operatorname{det}\left(A+\sum_{q=1}^{m} \xi_{q} C_{q}\right) \operatorname{det}\left(\lambda I_{d}-G(\xi)\right) .
\end{gathered}
$$

Condition (b) leads to

$$
\begin{array}{r}
P(\lambda)=\widehat{P}\left(\lambda, e^{-\lambda \tau_{1}}, e^{-\lambda \tau_{2}}, \ldots, e^{-\lambda \tau_{m}}\right) \neq 0 \\
\text { for } \operatorname{Re}(\lambda) \geq 0 .
\end{array}
$$

Hence

$$
\operatorname{Sup}\{\operatorname{Re}(\lambda): P(\lambda)=0\} \leq 0 .
$$

Now we will show that the strict inequality in (16) holds. Define

$$
F\left(\xi_{1}, \xi_{2}, \ldots, \xi_{m}\right)=\operatorname{det}\left(A+\sum_{q=1}^{m} \xi_{q} C_{q}\right),
$$

and then $F\left(\xi_{1}, \xi_{2}, \ldots, \xi_{m}\right)$ is a multivariate polynomial and is nonzero on the compact domain defined by $\left|\xi_{q}\right| \leq 1, q=$ $1,2, \ldots, m$, and equal to 1 at the origin. Hence, its modulus is bounded; that is,

$$
\begin{aligned}
& \quad\left|F\left(\xi_{1}, \xi_{2}, \ldots, \xi_{m}\right)\right| \geq \varepsilon>0, \\
& \text { when }\left|\xi_{q}\right| \leq 1, \quad \text { for } q=1,2, \ldots, m .
\end{aligned}
$$

By the continuity of $F$, there exists a $\delta>0$ such that

$$
\begin{aligned}
& \quad\left|F\left(\xi_{1}, \xi_{2}, \ldots, \xi_{m}\right)\right|>0, \\
& \text { when }\left|\xi_{q}\right| \leq 1+\delta, \quad \text { for } q=1,2, \ldots, m
\end{aligned}
$$

It follows from this that

$$
\begin{aligned}
& \operatorname{det}\left(A+\sum_{q=1}^{m} \xi_{q} C_{q}\right) \neq 0, \\
& \text { when }\left|e^{-\lambda \tau_{q}}\right| \leq 1+\delta, \quad \text { for } q=1,2, \ldots, m .
\end{aligned}
$$

Let $r$ be the strictly positive number $r=\ln (1+\delta) / \tau$; then

$$
\operatorname{det}\left(A+\sum_{q=1}^{m} e^{-\lambda \tau_{q}} C_{q}\right) \neq 0 \text { for } \operatorname{Re}(\lambda) \geq-r
$$

Thus, the equation $\widetilde{P}(\lambda)=0$ has only a finite number of roots when $\operatorname{Re}(\lambda) \geq-r$, and it holds true for the equation $p(\lambda)=0$ by condition (a). Combined with (16) we get that the characteristic equation has at most a finite number of roots in the region $\{\lambda:-r \leq \operatorname{Re}(\lambda)<0\}$.

Let

$$
-\gamma=\max _{-r \leq \operatorname{Re}(\lambda)<0}\{\operatorname{Re}(\lambda)\}
$$

then $\gamma>0$.

When $\operatorname{Re}(\lambda)>-\gamma$, the characteristic equation $p(\lambda)=$ 0 has no root. Hence, a strict inequality holds in (16). By Lemma 2, the proof is completed.

2.2. The Two-Step Runge-Kutta Methods and the Stability Region. Consider the two-step Runge-Kutta method:

$$
\begin{aligned}
& Y^{(n)}=h C_{11} F\left(t_{n}, Y^{(n)}\right)+C_{12} y^{(n-1)} \\
& y^{(n)}=h C_{21} F\left(t_{n}, Y^{(n)}\right)+C_{22} y^{(n-1)}
\end{aligned}
$$

for solving the initial value problem (1). 
In order to simplify the analysis, we consider two-step Runge-Kutta method (TSRK) of the form

$$
\begin{gathered}
u_{n+1}=(1-\theta) u_{n}+\theta u_{n-1} \\
+h \sum_{j=1}^{s} \widehat{b}_{j} f\left(t_{j}, U_{n}^{j}\right) \\
+h \sum_{j=1}^{s} \tilde{b}_{j} f\left(\tilde{t}_{j}, U_{n-1}^{j}\right), \\
U_{n}^{i}=u_{n}+h \sum_{j=1}^{s} \tilde{a}_{i, j} f\left(t_{j}, U_{n}^{j}\right) \quad i=1,2, \ldots, s,
\end{gathered}
$$

where $t_{j}=t_{n}+c_{j} h, \widetilde{t}_{j}=t_{n-1}+c_{j} h, u_{i}$ is an approximation to $u\left(t_{i}\right), h$ is a fixed step-size, $\theta, \widehat{b}_{j}, \widetilde{b}_{j}, \widetilde{a}_{i, j}$, and $c_{j}$ are coefficients of the method, $0 \leq \theta \leq 1$.

These methods are a subclass of general linear methods introduced by Butcher [15] and could be possibly also referred to as two-step hybrid methods. They generalize $k$-step collocation methods (with $k=2$ ) for ordinary differential equations (ODEs) studied by Lie and Nørsett [16] and Lie [17] and two-step Runge-Kutta methods for ODEs investigated by Byrne and Lambert [18]. The variable stepsize continuous two-step Runge-Kutta methods for ODEs were investigated by Jackiewicz and Tracogna [19]. Here we will represent (24a) and $(24 \mathrm{~b})$ by the following table of the coefficients:

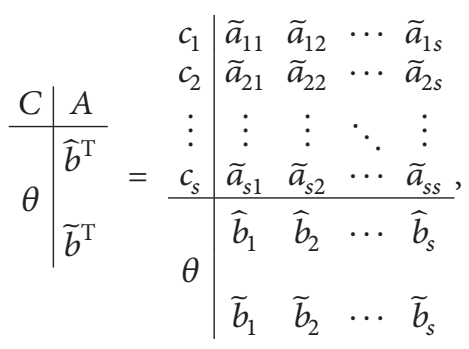

where $c_{i}=\sum_{j=1}^{s} \widetilde{a}_{i j}$ and $\sum_{j=1}^{s}\left(\widehat{b}_{j}+\widetilde{b}_{j}\right)=1+\theta$.

Apply (24a) and (24b) to the basic test equation

$$
u^{\prime}(t)=a u(t) \quad t \geq 0, \operatorname{Re} a<0,
$$

which gives the following equations:

$$
\begin{gathered}
u_{n+1}=(1-\theta) u_{n}+\theta u_{n-1}+\alpha\left(\sum_{j=1}^{s} \widehat{b}_{j} U_{n}^{j}+\widetilde{b}_{j} U_{n-1}^{j}\right) \\
U_{n}^{i}=u_{n}+\alpha \sum_{j=1}^{s} \widetilde{a}_{i, j} U_{n}^{j} .
\end{gathered}
$$

Rewriting (27) we obtain

$$
u_{i+1}=R(\alpha, \theta) u_{i}+S(\alpha, \theta) u_{i-1},
$$

where

$$
\begin{gathered}
R(\alpha, \theta)=1-\theta+\alpha \widehat{b}^{\mathrm{T}}(I-\alpha \widetilde{A})^{-1} e \\
S(\alpha, \theta)=\theta+\alpha \widetilde{b}^{\mathrm{T}}(I-\alpha \widetilde{A})^{-1} e \\
\alpha=a h, \quad e=[1,1, \ldots, 1]_{s}^{\mathrm{T}}
\end{gathered}
$$

To investigate the stability properties of (24a) and (24b) with (26), we must investigate the asymptotic behaviors of the solution to (28). This is determined by the location of roots of the characteristic polynomial

$$
\varphi(z)=z^{2}-R(a, \theta) z-S(a, \theta)
$$

The stability region of the two-step Runge-Kutta methods (24a) and (24b) is the set of all points $\alpha$ for which the roots of $\varphi(z)$ are inside or on the unit circle with those on the unit circle being simple. If $\varphi(z)$ is a Schur polynomial for any $\alpha$ with $\operatorname{Re} \alpha<0$, the stability of the two-step Runge-Kutta method contains the negative half plane; the method is said to be A-stable for ODEs.

\section{Asymptotic Stability of TSRK Methods for Neutral Delay Integro Differential-Algebraic Equation with Many Delays}

In this section, we will confine our discussion to neutral delay integro differential-algebraic equation with commensurate delays, that is, systems of the form (1) with $\tau_{q}=q \tau, \tau=M h$, $M$ is a positive integer, $q=1,2, \ldots, m$.

Definition 6 (see [20]). A numerical method for asymptotically stable system (1) is called asymptotically stable if the numerical solution satisfies

$$
\lim _{n \rightarrow \infty} u_{n}=0
$$

Applying the two-step method (24a) and (24b) to (1), we have

$$
u_{n+1}=(1-\theta) u_{n}+\theta u_{n-1}+\sum_{j=1}^{s} \widehat{b}_{j} K_{n, j}+\sum_{j=1}^{s} \widetilde{b}_{j} K_{n-1, j}
$$

$$
A K_{n, i}+h B\left(u_{n}+\sum_{j=1}^{s} \tilde{a}_{i j} k_{n, j}\right)+\sum_{q=1}^{m} C_{q} K_{n-q M, i}
$$

$$
\begin{array}{r}
+h \sum_{q=1}^{m} D_{q}\left(u_{n-q M}+\sum_{j=1}^{s} \tilde{a}_{i j} K_{n-q M, j}\right) \\
+h \sum_{q=1}^{m} \sum_{\gamma=0}^{q M} \gamma_{r} G_{q} \cdot\left(u_{n-r}+\sum_{j=1}^{s} \tilde{a}_{i j} k_{n-r, j}\right)=0 \\
\text { for } i=1,2, \ldots, s,
\end{array}
$$

where $K_{n, i}=\left[K_{n, i}^{1}, K_{n, i}^{2}, \ldots, K_{n, i}^{d}\right]^{\mathrm{T}}, i=1,2, \ldots, s$, are stage derivatives multiplied by $h$.

Let

$$
\begin{gathered}
\widehat{b}^{\mathrm{T}}=\left[\widehat{b}_{1}, \widehat{b}_{2}, \ldots, \widehat{b}_{s}\right], \quad \tilde{b}^{\mathrm{T}}=\left[\widetilde{b}_{1}, \widetilde{b}_{2}, \ldots, \widetilde{b}_{s}\right], \\
\widetilde{A}=\left(\widetilde{a}_{i j}\right) .
\end{gathered}
$$


We assume that all the eigenvalues of $\widetilde{A}$ have positive real part. Rearrange the variables of the stage derivatives as

$$
\begin{aligned}
K_{n}=[ & K_{n, 1}^{1}, K_{n, 2}^{1}, \ldots, K_{n, s}^{1}, K_{n, 1}^{2}, K_{n, 2}^{2}, \\
& \left.\ldots, K_{n, s}^{2}, \ldots, K_{n, 1}^{d}, K_{n, 2}^{d}, \ldots, K_{n, s}^{d}\right]^{\mathrm{T}} .
\end{aligned}
$$

Define

$$
\begin{gathered}
Y_{n}=\left(K_{n}^{\mathrm{T}}, u_{n+1}^{\mathrm{T}}\right)^{\mathrm{T}}, \quad \bar{B}=h B, \quad \bar{D}_{q}=h D_{q}, \\
\overline{\bar{G}}_{q}=h^{2} G_{q} .
\end{gathered}
$$

Rewrite (32) and (33) in the form

$$
\begin{aligned}
& {\left[\begin{array}{cc}
A \otimes I_{s}+\bar{B} \otimes \widetilde{A} & 0 \\
-I_{d} \otimes \widehat{b}^{T} & I_{d}
\end{array}\right] Y_{n}} \\
& +\left[\begin{array}{cc}
0 & \bar{B} \otimes e \\
-I_{d} \otimes \tilde{b}^{\mathrm{T}} & -(1-\theta) I_{d}
\end{array}\right] Y_{n-1} \\
& +\left[\begin{array}{cc}
0 & 0 \\
0 & -\theta I_{d}
\end{array}\right] Y_{n-2} \\
& +\sum_{q=1}^{m}\left[\begin{array}{ccc}
C_{q} \otimes I_{s}+\bar{D}_{q} \otimes \widetilde{A} & 0 \\
0 & 0
\end{array}\right] Y_{n-q M} \\
& +\sum_{q=1}^{m}\left[\begin{array}{cc}
0 & \bar{D}_{q} \otimes e \\
0 & 0
\end{array}\right] Y_{n-q M-1} \\
& +\sum_{q=1}^{m} \sum_{r=0}^{M q}\left[\begin{array}{cr}
C_{q} \otimes I_{s}+\bar{D}_{q} \otimes \widetilde{A} & 0 \\
0 & 0
\end{array}\right] Y_{n-r} \\
& +\sum_{q=1}^{m} \sum_{r=0}^{M q}\left[\begin{array}{cc}
0 & \gamma_{r} \overline{\bar{G}}_{q} \otimes e \\
0 & 0
\end{array}\right] Y_{n-r-1}=0 .
\end{aligned}
$$

The characteristic polynomial of (37) is given by

$$
p(z)=\operatorname{det}\left[\begin{array}{ll}
T_{1}(z) & T_{2}(z) \\
T_{3}(z) & T_{4}(z)
\end{array}\right], \quad z \in C,
$$

where

$$
\begin{aligned}
T_{1}(z)=z^{2}[ & \left(A \otimes I_{s}+\bar{B} \otimes \widetilde{A}\right) \\
& +\sum_{q=1}^{m}\left(C_{q} \otimes I_{s}+\bar{D}_{q} \otimes \widetilde{A}\right) z^{-q M} \\
& \left.+\sum_{q=1}^{m} \sum_{r=0}^{M q} \gamma_{r} \overline{\bar{G}}_{q} \otimes \widetilde{A} z^{-r}\right],
\end{aligned}
$$

$$
\begin{array}{r}
T_{2}(z)=z\left[\bar{B} \otimes e+\sum_{q=1}^{m} \bar{D}_{q} \otimes e z^{-q M}\right. \\
\left.+\sum_{q=1}^{m} \sum_{r=0}^{M q} \gamma_{r} \overline{\bar{G}}_{q} \otimes e r^{-r}\right], \\
T_{3}(z)=-z^{2} I_{d} \otimes \widehat{b}^{\mathrm{T}}-z I_{d} \otimes \widetilde{b}^{\mathrm{T}}, \\
T_{4}(z)=z^{2} I_{d}-z(1-\theta) I_{d}-\theta I_{d} .
\end{array}
$$

Following from the theorem on difference equations, we get that if all the zeros $z$ of (38) satisfy $|z|<1$, then

$$
\lim _{n \rightarrow \infty} Y_{n}=0 \text {. }
$$

Hence, we formulate the following lemmas.

Lemma 7 (see [21]). If all the zeros $z$ of (38) satisfy $|z|<1$, then numerical method ((32) and (33)) satisfies

$$
\lim _{n \rightarrow \infty} u_{n}=0 \text {. }
$$

Lemma 8. Assume that condition (a) of Theorem 5 holds and assume that $\left[I_{s}-\lambda_{l}(r(z)) \widetilde{A}\right]$ are invertible for $|z| \geq 1$, where

$$
\begin{aligned}
r(z)= & \left(A+\sum_{q=1}^{m} z^{-q M} C_{q}\right)^{-1} \\
& \times\left(-\bar{B}-\sum_{q=1}^{m} z^{-q M} \bar{D}_{q}-\sum_{q=1}^{m} \sum_{r=0}^{q M} \gamma_{r} z^{-r} \overline{\bar{G}}_{q}\right) ;
\end{aligned}
$$

then, $\operatorname{det}\left[T_{1}(z)\right] \neq 0$, for $|z| \geq 1$.

Proof. Condition (a) in Theorem 5 implies that the matrix $\left(A+\sum_{q=1}^{m} z^{-q M} C_{q}\right)$ is invertible for $|z| \geq 1$; then $T_{1}(z)=$ $z^{2}\left[\left(A+\sum_{q=1}^{m} z^{-q M} C_{q}\right) \otimes I_{s}\right]\left[I_{d} \otimes I_{s}-r(z) \otimes \widetilde{A}\right]$.

We have that

$$
\begin{aligned}
\operatorname{det}\left[T_{1}(z)\right]= & z^{2 s d}\left[\operatorname{det}\left(A+\sum_{q=1}^{m} z^{-q M} C_{q}\right)\right]^{s} \\
& \times \operatorname{det}\left[I_{d} \otimes I_{s}-r(z) \otimes \widetilde{A}\right] \\
= & z^{2 s d}\left[\operatorname{det}\left(A+\sum_{q=1}^{m} z^{-q M} C_{q}\right)\right]^{s} \\
& \times \prod_{l=1}^{d} \coprod_{j=1}^{s}\left[1-\lambda_{l}(r(z)) \lambda_{j}(\widetilde{A})\right] .
\end{aligned}
$$

The matrix $I_{s}-\lambda_{l}(r(z)) \widetilde{A}$ is invertible meaning that $\lambda_{l}(r(z)) \lambda_{j}(\widetilde{A}) \neq 1$ for all $l, j$.

Hence, $\operatorname{det}\left[T_{1}(z)\right] \neq 0$, for $|z| \geq 1$.

Theorem 9. If the system ((32) and (33)) satisfies Lemma 8 and the following conditions, 


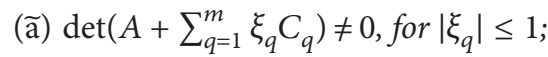

( $\widetilde{\mathrm{b}}) \mid 1-\theta-z^{-1} \theta-\left(\widehat{b}^{\mathrm{T}}+z^{-1} \widetilde{b}^{\mathrm{T}}\right)\left[I_{d} \otimes I_{s}-r(z) \otimes \widetilde{A}\right]^{-1}\left[e_{s} \otimes\right.$ $r(z)] \mid<1$, for $|z| \geq 1$,

then the solution of the TSRK methods for (1) is asymptotically stable.

Proof. By Lemma 7, we need to prove that all the zeros of (38) satisfy $|z|<1$.

If these were not true, there would exist a $z_{0} \in C$ with $\left|z_{0}\right| \geq 1$, such that

$$
\operatorname{det}\left[\begin{array}{ll}
T_{1}\left(z_{0}\right) & T_{2}\left(z_{0}\right) \\
T_{3}\left(z_{0}\right) & T_{4}\left(z_{0}\right)
\end{array}\right]=0 .
$$

By Lemma 8 , we have that $\operatorname{det}\left[T_{1}\left(z_{0}\right)\right] \neq 0$.

Hence, (44) is equivalent to

$$
\operatorname{det}\left[T_{4}\left(z_{0}\right)-T_{3}\left(z_{0}\right) T_{1}^{-1}\left(z_{0}\right) T_{2}\left(z_{0}\right)\right\rfloor=0 .
$$

Using the Kronecker product [5, chapter 4], we have that

$$
\begin{aligned}
& \operatorname{det}\left[T_{4}\left(z_{0}\right)-T_{3}\left(z_{0}\right) T_{1}^{-1}\left(z_{0}\right) T_{2}\left(z_{0}\right)\right\rfloor \\
& =\operatorname{det}\left\{z_{0}^{2} I_{d}-z_{0}(1-\theta) I_{d}-\theta I_{d}\right. \\
& -\left(z_{0}^{2} I_{d} \otimes \widehat{b}^{\mathrm{T}}+z_{0} I_{d} \otimes \widetilde{b}^{\mathrm{T}}\right) \\
& \times z_{0}^{-2}\left[\left(A+\sum_{q=1}^{m} z_{0}^{-q M} C_{q}\right) \otimes I_{s}\right]^{-1} \\
& \cdot\left[I_{d} \otimes I_{s}-r\left(z_{0}\right) \otimes \widetilde{A}\right]^{-1} \\
& \cdot z_{0}\left[\bar{B} \otimes e+\sum_{q=1}^{m} \bar{D}_{q} \otimes e z_{0}^{-q M}\right. \\
& \left.\left.+\sum_{q=1}^{m} \sum_{r=0}^{M q} \gamma_{r} \overline{\bar{G}}_{q} \otimes e z_{0}^{-r}\right]\right\} \\
& =z_{0}^{d} \operatorname{det}\left\{z_{0} I_{d}-\left[(1-\theta) I_{d}-z_{0}^{-1} \theta I_{d}\right.\right. \\
& -\left(I_{d} \otimes \widehat{b}^{\mathrm{T}}+z_{0}^{-1} I_{d} \otimes \widetilde{b}^{\mathrm{T}}\right) \\
& \times\left[I_{d} \otimes I_{s}-r\left(z_{0}\right) \otimes \widetilde{A}\right]^{-1} \\
& \left.\left.\times\left[e \otimes r\left(z_{0}\right)\right]\right]\right\} \text {. }
\end{aligned}
$$

Combining (45) and (46) gives that

$$
\begin{aligned}
\operatorname{det}\left\{z_{0} I_{d}-[\right. & (1-\theta) I_{d}-z_{0}^{-1} \theta I_{d} \\
& -\left(I_{d} \otimes \widehat{b}^{\mathrm{T}}+z_{0}^{-1} I_{d} \otimes \widetilde{b}^{\mathrm{T}}\right) \\
& \left.\left.\cdot\left[I_{d} \otimes I_{s}-r\left(z_{0}\right) \otimes \widetilde{A}\right]^{-1}\left[e \otimes r\left(z_{0}\right)\right]\right]\right\}=0,
\end{aligned}
$$

which implies $\mid 1-\theta-z_{0}^{-1} \theta-\left(\widehat{b}^{\mathrm{T}}+z_{0}^{-1} \widetilde{b}^{\mathrm{T}}\right)\left[I_{d} \otimes I_{s}-r\left(z_{0}\right) \otimes \widetilde{A}\right]^{-1}$ $\left[e \otimes r\left(z_{0}\right)\right] \mid \geq 1$.

This contradicts the assumption that $(\widetilde{b})$ for $\left|z_{0}\right| \geq 1$. Hence, the theorem is proved.

\section{Numerical Experiments}

Example 1. Consider the following linear system:

$$
\begin{aligned}
& A u^{\prime}(t)+B u(t)+\sum_{q=1}^{m} C_{q} u^{\prime}\left(t-\tau_{q}\right) \\
& +\sum_{q=1}^{m} D_{q} u\left(t-\tau_{q}\right) \\
& +\sum_{q=1}^{m} G_{q} \int_{t-\tau_{q}}^{t} u(\delta) d \delta=0, \quad t \geq 0, \\
& u(t)=\varphi(t), \quad t \in[-\tau, 0),
\end{aligned}
$$

with $m=2, \tau_{1}=1, \tau_{2}=2$, and $\varphi(t)=(\cos (t), \sin (t), \cos (t))^{\mathrm{T}}$ for $t \in[-2,0]$, where

$$
\begin{array}{ccc}
A=\left[\begin{array}{lll}
1 & 0 & 0 \\
0 & 0 & 0 \\
0 & 1 & 0
\end{array}\right], & B=\left[\begin{array}{ccc}
1.5 & 0 & 0 \\
0 & 0 & -0.4 \\
0 & 0.8 & 0.5
\end{array}\right], \\
D_{1}=\left[\begin{array}{ccc}
0.2 & 0.4 & 0 \\
0 & 0 & 0.1 \\
0 & 0.2 & -0.2
\end{array}\right], & D_{2}=\left[\begin{array}{ccc}
0.1 & 0 & 0 \\
0 & 0 & 0.1 \\
0 & 0.15 & 0.8
\end{array}\right], \\
G_{1}=\left[\begin{array}{ccc}
0.2 & 0 & 0 \\
0 & 0 & -0.2 \\
0 & 0.1 & 0.25
\end{array}\right], & G_{2}=\left[\begin{array}{ccc}
0.1 & 0 & 0 \\
0 & 0 & -0.2 \\
0 & 0.05 & 0.25
\end{array}\right],
\end{array}
$$

and $C_{1}=-0.3 A$ and $C_{2}=-0.5 A$.

Here the matrix coefficients satisfy Theorem 9. Hence, the system is asymptotically stable.

We choose the A-stable TSRK methods as follows [22]:

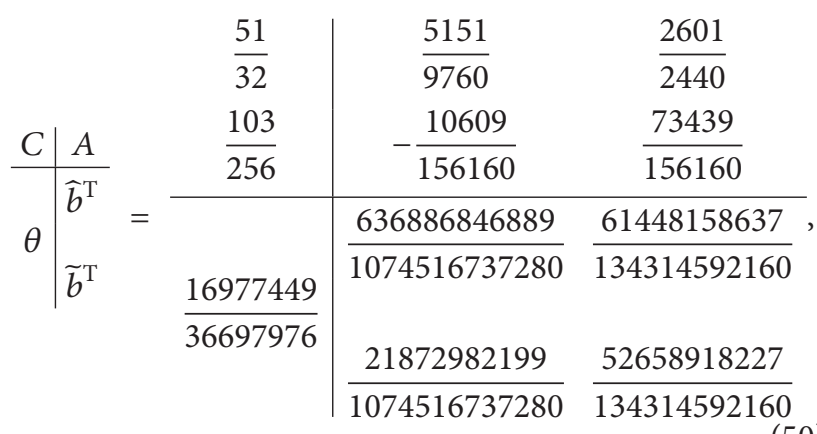

where

$$
c_{i}=\sum_{j=1}^{s} \tilde{a}_{i j}, \quad \sum_{j=1}^{s}\left(\widehat{b}_{j}+\widetilde{b}_{j}\right)=1+\theta .
$$


It can be easily seen that the A-stable TSRK method is asymptotically stable, which illuminates the conclusion of Theorem 9.

\section{Conclusions}

This paper develops the asymptotic stability of the two-step Runge-Kutta methods for neutral delay integro differentialalgebraic equations with many delays. It studies the asymptotic stability of the analytical solution and introduces two step Runge-Kutta methods and the stability region. It also deals with the asymptotic stability of two-step RungeKutta method for neutral delay integro differential-algebraic equations with many delays and proves that the A-stable two-step Runge-Kutta methods are asymptotically stable for neutral delay integro differential-algebraic equations with many delays.

\section{Conflict of Interests}

The authors declare that there is no conflict of interests regarding the publication of this paper.

\section{Acknowledgment}

This work was supported by the Doctor Foundation of Heilongjiang Institute of Technology (2012BJ27).

\section{References}

[1] K. E. Brenan, S. L. Campbell, and L. R. Petzold, Numerical Solution of Initial-Value Problem in Differential-Algebraic Equations, SIAM, Philadephia, Pa, USA, 2nd edition, 1995.

[2] E. Hairer, S. P. Norsett, and G. Wanner, Solving Ordinary Differential-Algebraic Equations $q$ Stiff and Differential-Algebraic Problems, Springer, New York, NY, USA, 1992.

[3] L. R. Petzold, Numerical Solutions of Differential-Algebraic Equations, Clarendon Press, Oxford, UK, 1995.

[4] U. M. Ascher and L. R. Petzold, "The numerical solution of delay-differential-algebraic equations of retarded and neutral type," SIAM Journal on Numerical Analysis, vol. 32, no. 5, pp. 1635-1657, 1995.

[5] S. L. Campbell, "Singular linear systems of differential equations with delays," Applicable Analysis, vol. 11, no. 2, pp. 129-136, 1980.

[6] R. K. Brayton and R. A. Willoughby, "On the numerical integration of a symmetric system of difference-differential equations of neutral type," Journal of Mathematical Analysis and Applications, vol. 18, pp. 182-189, 1967.

[7] W. Zhu and L. R. Petzold, "Asymptotic stability of linear delay differential-algebraic equations and numerical methods," Applied Numerical Mathematics, vol. 24, no. 2-3, pp. 247-264, 1997.

[8] J. J. Zhao, Y. Xu, S. Y. Dong, and M. Z. Liu, "Stability of the Rosenbrock methods for the neutral delay differential-algebraic equations," Applied Mathematics and Computation, vol. 168, no. 2, pp. 1128-1144, 2005.

[9] Q. H. Yu, L. P. Sun, and Y. Li, "Asymptotic stability of linear multistep methods for generalized neutral delay differentialalgebraic equations," Journal of System Simulation, vol. 21, no. 20, pp. 6432-6435, 2009.
[10] C. T. H. Baker and N. J. Ford, "Stability properties of a scheme for the approximate solution of a delay-integro-differential equation," Applied Numerical Mathematics, vol. 9, no. 3-5, pp. 357-370, 1992.

[11] T. Koto, "Stability of Runge-Kutta methods for delay integrodifferential equations," Journal of Computational and Applied Mathematics, vol. 145, no. 2, pp. 483-492, 2002.

[12] C. Huang and S. Vandewalle, "An analysis of delay-dependent stability for ordinary and partial differential equations with fixed and distributed delays," SIAM Journal on Scientific Computing, vol. 25, no. 5, pp. 1608-1632, 2004.

[13] T. Luzyanina, K. Engelborghs, and D. Roose, "Computing stability of differential equations with bounded distributed delays," Numerical Algorithms, vol. 34, no. 1, pp. 41-66, 2003.

[14] C. J. Zhang and S. F. Vandewalle, "Stability criteria for exact and discrete solutions of neutral multidelay-integro-differential equations," Advances in Computational Mathematics, vol. 28, no. 4, pp. 383-399, 2008.

[15] J. C. Butcher, The Numerical Analysis of Ordinary Differential Equations, John Wiley \& Sons, Chichester, UK, 1987.

[16] I. Lie and S. P. Nørsett, "Superconvergence for multistep collocation," Mathematics of Computation, vol. 52, no. 185, pp. 65-79, 1989.

[17] I. Lie, "Local error estimation for multistep collocation methods," Numerical Mathematics, vol. 30, no. 1, pp. 126-144, 1990.

[18] G. D. Byrne and R. J. Lambert, "Pseudo-Runge-Kutta methods involving two points," Journal of the Association for Computing Machinery, vol. 13, pp. 114-123, 1966.

[19] Z. Jackiewicz and S. Tracogna, "Variable stepsize continuous two-step Runge-Kutta methods for ordinary differential equations," Numerical Algorithms, vol. 12, no. 3-4, pp. 347-368, 1996.

[20] Q. Wang, Y. H. Cong, and J. J. Li, "Stability of two-step RungeKutta methods for neutral delay-integro-differential-algebraic equations," Journal of Shanghai Normal University: Natural Sciences, vol. 39, no. 6, pp. 551-557, 2010.

[21] P. Hu and C. Huang, "Analytical and numerical stability of nonlinear neutral delay integro-differential equations," Journal of the Franklin Institute: Engineering and Applied Mathematics, vol. 348, no. 6, pp. 1082-1100, 2011.

[22] Z. Jackiewicz, R. Renaut, and A. Feldstein, "Two-step RungeKutta methods," SIAM Journal on Numerical Analysis, vol. 28, no. 4, pp. 1165-1182, 1991. 


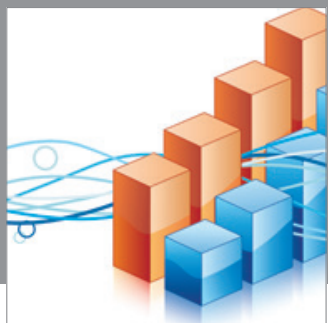

Advances in

Operations Research

mansans

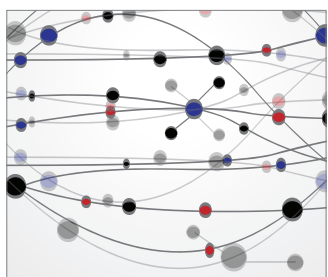

The Scientific World Journal
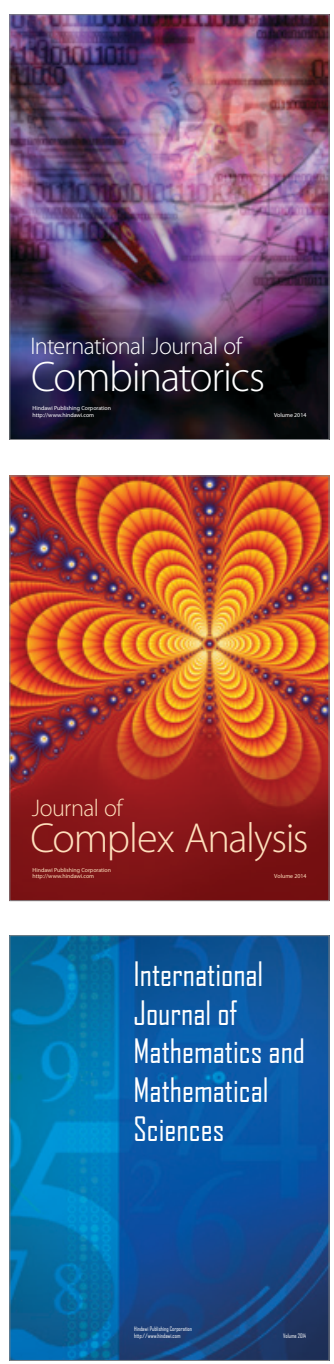
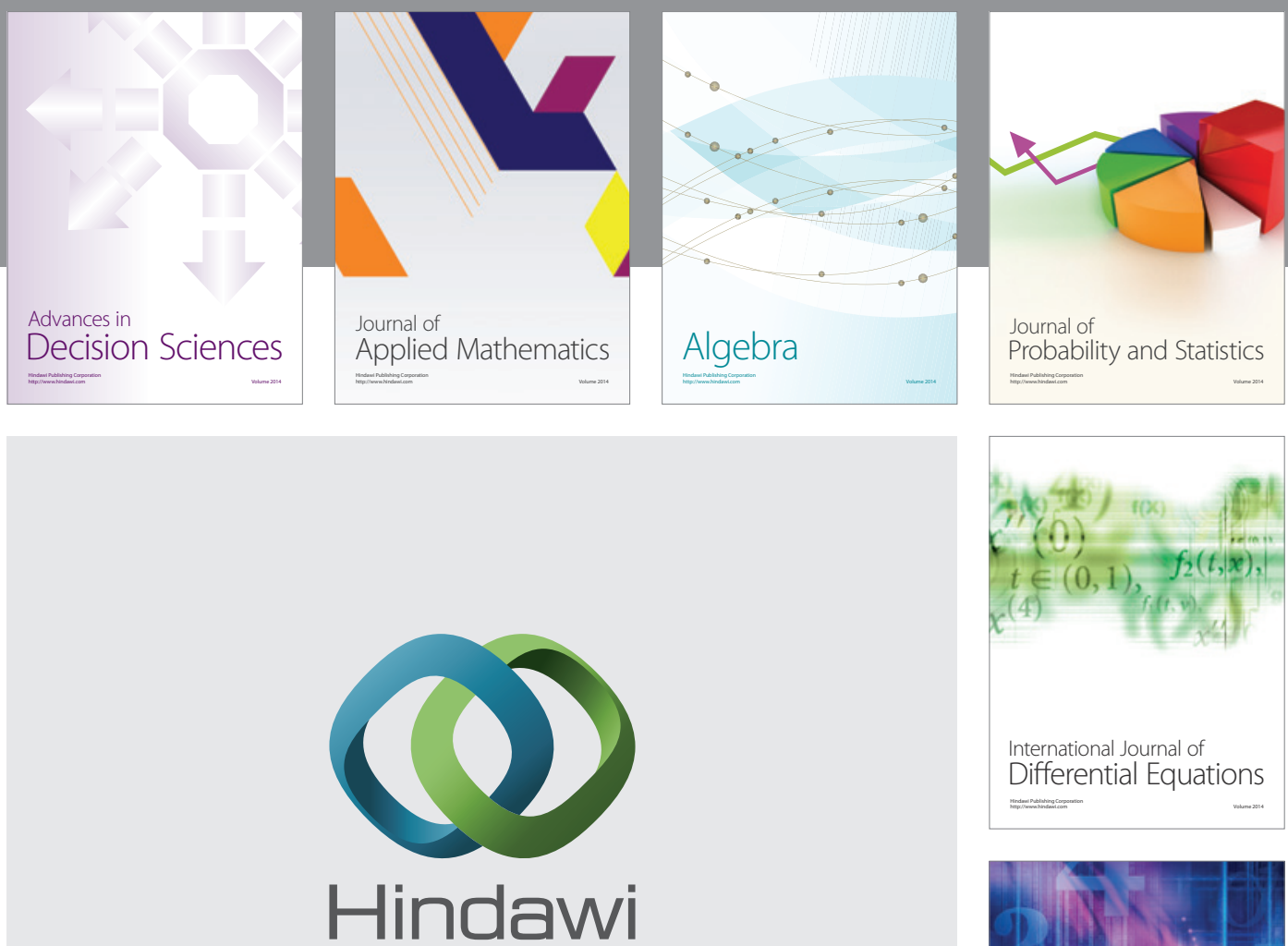

Submit your manuscripts at http://www.hindawi.com
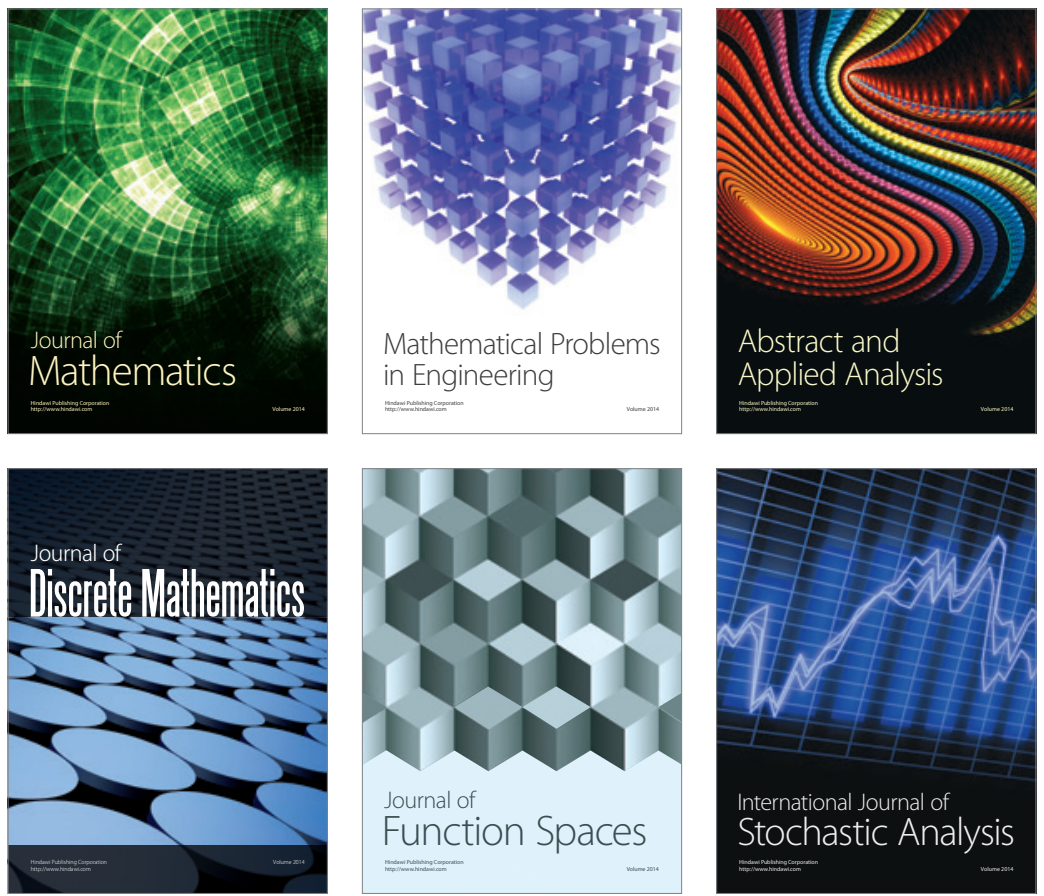

Journal of

Function Spaces

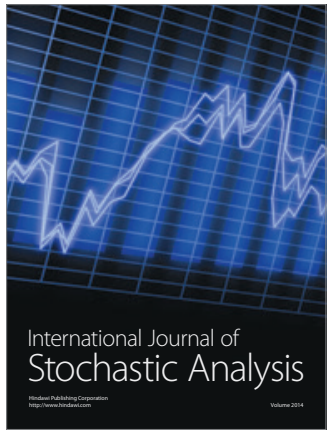

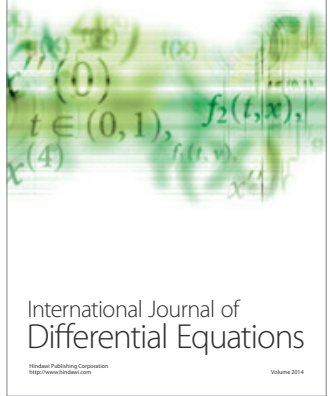
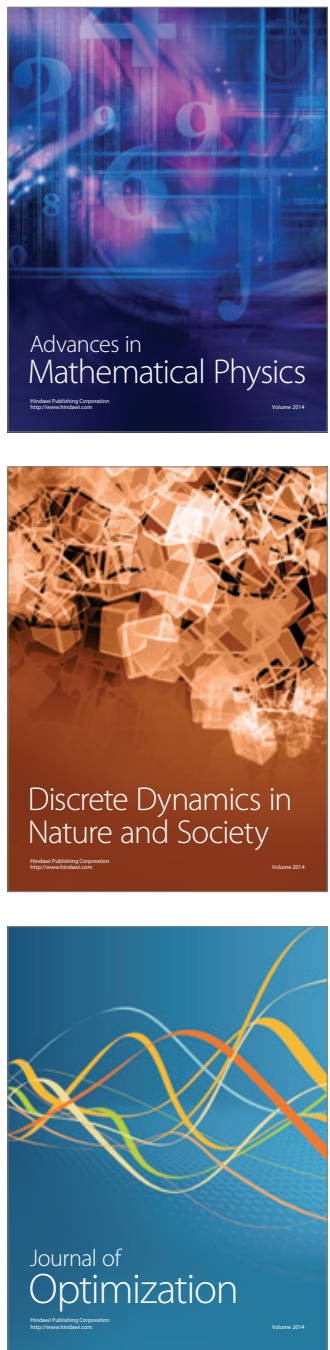